\title{
Tratamiento a largo plazo del dolor crónico con opioides: certeza sobre sus riesgos e incerteza sobre su efectividad
}

Long-term treatment of chronic pain with opioids: certain risks and uncertain effectiveness

\section{Objetivos}

Evaluar la efectividad y los riesgos del tratamiento a largo plazo (mayor de tres meses) del dolor crónico con opioides.

\section{Diseño}

Revisión sistemática.

Fuente de datos y selección de estudios

La búsqueda se realizó en MEDLINE, el Registro Cochrane CENTRAL de Ensayos Controlados, la Base de Datos Cochrane de Revisiones Sistemáticas, PsycINFO, CINAHL (enero 2008 a agosto 2014), y en el registro de ensayos ClinicalTrials.gov. Además se revisaron las listas de referencias de revisiones previas y de los artículos relevantes identificados.

Se incluyeron ensayos clínicos aleatorizados y estudios observacionales de adultos con dolor crónico en tratamiento a largo plazo con opiáceos comparado con placebo, ningún opioide u otra terapia no opioide, y estudios que evaluaran diferentes estrategias de dosificación de opioides o de mitigación de riesgos. Se restringió la inclusión a estudios que estuvieran publicados en inglés. Dos revisores seleccionaron, extractaron y valoraron la calidad de los estudios de forma independiente.

\section{Resultados}

De las 4.209 referencias identificadas finalmente se incluyeron 39 estudios. Ningún ensayo controlado con placebo cumplió con los criterios de selección, por lo que la evidencia fue escasa para muchas comparaciones y resultados. No se encontraron artículos de más de un año de seguimiento que demostraran beneficios, incluyendo mejoría funcional y calidad de vida, del tratamiento con opioides frente a otro tratamiento. Se encontró asociación entre el tratamiento a largo plazo con opioides y riesgo de fracturas, infarto agudo de miocardio, disfunción sexual, sobredosificación y adicción. En un estudio las tasas de abuso de opiáceos o dependencia fue $0,7 \%$ con dosis bajas y $6,1 \%$ con dosis altas: OR 14,9 (IC95\% 10,4 a 21,5) y 122,5 (IC95\% 72,8 a 206,0) respectivamente, en comparación con tratamiento no opioide. En otros estudios la prevalencia de dependencia osciló entre 3 y $26 \%$.

Un gran estudio de cohorte retrospectiva encontró que el uso reciente de opioides se asoció con un mayor riesgo de eventos serios de sobredosis: HR 8,4 IC95\% 2,5 a 28.

Si bien se encontraron varios artículos en los que se evaluó la metadona, no se determinó a largo plazo que hubiese diferencias significativas con el sulfato de morfina respecto a la mortalidad ni la implicancia de la prolongación del intervalo QT. No se encontró una estrategia segura y efectiva en relación a la titulación ni diferencia entre los opioides de liberación prolongada comparado con los de rápida acción.

\section{Conclusión}

No existe evidencia de beneficio a largo plazo de los opioides para el dolor crónico, pero sí de eventos adversos serios observándose un efecto dosis-respuesta.

\section{Comentario}

Si bien hace más de diez años los especialistas de dolor preconizamos la necesidad de escalar opioides en pacientes con dolor oncológico, y en consecuencia, el uso de los mismos fue en aumento ${ }^{1-3}$, es posible que no hayamos puesto el límite a tiempo en pacientes con dolor crónico no oncológico.

Durante muchos años se mantuvieron pacientes con dosis altas de opioides minimizando sus efectos colaterales y con bastante poco nivel de alarma respecto de su trasfondo psiquiátrico y el daño potencial que le podría generar rotarlo a un opioide potente.

El problema no solo está presente cuando se utiliza la vía oral, sino principalmente en pacientes con bomba de infusión espinal. Si se utiliza este tipo de drogas en personas con trastornos de la personalidad, aumenta el riesgo de sobredosis. También en cierta medida se ha abusado de indicaciones intervencionistas como estimuladores medulares. Posiblemente los especialistas en tratamiento del dolor hayamos tenido estas conductas por la omnipotente imperiosidad de querer aliviar este síntoma sin terminar de entender al enfermo que lo padece. Debemos ser cuidadosos y plantearnos junto al paciente todas las posibilidades tanto coadyuvantes como intervencionistas, y si decidimos indicar un opioide potente, utilizar la mínima dosis efectiva asociada a coadyuvantes, pensando en todas las alternativas terapéuticas posibles. Si en seis meses no viéramos un claro beneficio, deberíamos considerar retirarlo. Es de buena práctica interconsultar a psiquiatría y pedir un psicodiagnóstico en el contexto de un paciente con dolor crónico, antes de rotar a un opioide potente. Esto no significa abandono, sino mayor cuidado. También resulta aconsejable mejorar la documentación de los efectos adversos de los tratamientos y de los fracasos terapéuticos. Esta revisión sistemática presenta como limitaciones la exclusión de estudios que no fueran en inglés, la imposibilidad de realizar meta-análisis y de evaluar sesgo de publicación, la ausencia de ensayos controlados con placebo y de estudios observacionales con adecuado control de potenciales factores de confusión y, especialmente, la ausencia de estudios con seguimiento mayor de un año. Aun así, resume en buena medida la evidencia relevante disponible sobre el tema.

\section{Conclusión del comentador}

Si bien aumentó la prescripción de opioides, hacen faltan más estudios para determinar la eficacia y los riesgos de la terapéutica con estas drogas a largo plazo en lo que respecta a dolor crónico no oncológico. Nada más fácil que indicar un opioide potente y nada más difícil que retirarlo. La omnipotencia médica quizás sea nuestro peor enemigo.

Rodrigo Javier Agejas [ Sección Tratamiento del Dolor y Cuidados Paliativos del Servicio de Anestesia del Hospital Italiano de Buenos Aires rodrigo.agejas@ hospitalitaliano.org.ar]

Agejas RJ. Tratamiento a largo plazo del dolor crónico con opioides: certeza sobre sus riesgos e incerteza sobre su efectividad. Evid Act Pract Ambul. 2016;19(2):43. Comentado de: Chou R, y col. The effectiveness and risks of long-term opioid therapy for chronic pain: a systematic review for a National Institutes of Health Pathways to Prevention Workshop. Ann Intern Med. 2015;162(4):276-86. PMID: 25581257.

Referencias

1. Sullivan MD, y col. Trends in use of opioids for non-cancer pain conditions $2000-2005$ in commercial and Medicaid insurance plans: the TROUP study. Pain. 2008;138:4409. [PMID: 18547726] doi:10.1016/j.pain .2008.04.027

2. Boudreau D, y col. Trends in long-term opioid therapy for chronic non-cancer pain. Pharmacoepidemiol Drug Saf. 2009;18:1166-75. [PMID: 19718704] doi:10.1002/pds.1833 3. Olsen Y, y col. Opioid prescriptions by U.S. primary care physicians from 1992 to 2001. J Pain. 2006;7:225-35. [PMID: 16618466] 